\title{
Transtorno de Estresse Pós-Traumático: evolução dos critérios diagnósticos e prevalência
}

\author{
Gabriela Sbardelloto - Pontifícia Universidade Católica do Rio Grande do Sul, Porto Alegre, Brasil \\ Luiziana Souto Schaefer - Pontifícia Universidade Católica do Rio Grande do Sul, Porto Alegre, Brasil \\ Alice Reunsaat Justo - Pontifícia Universidade Católica do Rio Grande do Sul, Porto Alegre, Brasil \\ Christian Haag Kristensen 1 - Pontifícia Universidade Católica do Rio Grande do Sul, Porto Alegre, Brasil
}

\begin{abstract}
Resumo
O Transtorno de Estresse Pós-Traumático (TEPT) se caracteriza por sintomas persistentes de revivência, evitação e entorpecimento, e excitabilidade aumentada, após a exposição a um evento traumático. Estudos epidemiológicos mostram que, entre os transtornos de ansiedade, o TEPT é o terceiro mais prevalente. Desde 1980, quando foi reconhecido como um diagnóstico válido, mudanças conceituais importantes ocorreram. A partir disso, o presente artigo apresenta uma revisão crítica da literatura sobre o desenvolvimento dos critérios diagnósticos do TEPT ao longo das edições do Manual Diagnóstico e Estatístico dos Transtornos Mentais (DSM), bem como informações sobre a prevalência do transtorno.

Palavras-chave: Transtorno de Estresse Pós-Traumático, Diagnóstico, Prevalência.
\end{abstract}

Posttraumatic Stress Disorder: development of diagnostic criteria and prevalence

\begin{abstract}
Posttraumatic Stress Disorder (PTSD) can be characterized by persistent symptoms of reexperience, avoidance and numbing, and increased arousal following exposure to a traumatic event. Epidemiological studies showed that PTSD is the third most prevalent anxiety disorder. Since it's recognition in 1980 as a valid diagnosis, important conceptual changes occurred. This article presents a critical review of the literature regarding development of diagnosis criteria of PTSD in the Diagnostic and Statistical Manual of Mental Disorders (DSM) as well as information regarding its prevalence.

Keywords: Post-Traumatic Stress Disorder, Diagnosis, Prevalence
\end{abstract}

O Transtorno de Estresse Pós-Traumático (TEPT), caracterizado basicamente como um conjunto de sintomas envolvendo altos níveis de ansiedade e momentos em que o indivíduo tem a impressão de estar revivendo o evento traumático experienciado, é um transtorno bastante prevalente, atingindo cerca de $6,8 \%$ da população geral (Kessler, Berglund e colaboradores, 2005). As primeiras observações realizadas sobre os seus efeitos foram registradas a partir de estudos com veteranos de guerra (Schestatsky, Shansis, Ceitlin, Abreu \& Hauck, 2003; Sher, 2004). Posteriormente, os critérios diagnósticos que caracterizavam o TEPT foram modificados ao longo das diferentes edições do Manual Diagnóstico e Estatístico dos Transtornos Mentais (DSM; American Psychiatric Association [APA], 1980; 1987; 1994; 2002) numa tentativa de melhor compreender as peculiaridades do transtorno bem como de diferenciálo de outros transtornos possíveis, como por exemplo, o Transtorno de Estresse Agudo (TEA).

Embora eventos traumáticos como guerras não sejam mais tão presentes, atualmente, outras

\footnotetext{
${ }^{1}$ Endereço para correspondência:

Programa de Pós-Graduação em Psicologia

Pontifícia Universidade Católica do Rio Grande do Sul

Av. Ipiranga, 6681, Prédio 11, Sala 933. Porto Alegre, RS

CEP 90619-900.

E-mail: christian.kristensen@pucrs.br

Apoio Financeiro CAPES
}

situações comumente observadas também podem desencadear o TEPT, como assaltos, sequestros, tempestades, entre outras (APA, 2002). A alta prevalência do transtorno bem como os prejuízos e as implicações clínicas, sociais e econômicas que afetam os indivíduos que vivenciam um evento estressor traumático, desencadeando o TEPT, apontam para a importância de estudos que abordem esse transtorno. A partir disso, este artigo apresenta uma revisão crítica da literatura sobre os critérios diagnósticos e a prevalência do TEPT, considerando as mudanças em termos de diagnóstico ao longo das edições do Manual Diagnóstico e Estatístico dos Transtornos Mentais (DSM) e apontando alguns estudos epidemiológicos realizados, a fim de clarificar, contextualizar e dimensionar a amplitude do transtorno.

\section{Eventos Estressores, Trauma e Estresse}

Os eventos estressores apontados como mais favoráveis para o desenvolvimento de situações traumáticas podem ser categorizados em três grandes grupos: eventos intencionais provocados pelo homem, eventos não-intencionais provocados pelo homem e eventos provocados pela natureza (Schiraldi, 1999). No primeiro grupo, entre os eventos intencionais provocados pelo homem, podemos citar: guerra civil, incesto, estupro, sedução, tortura física ou emocional, assalto, crime violento sofrido pela própria pessoa ou por pessoas afetivamente significativas, terrorismo, participação em atrocidades violentas, alcoolismo e uso 
de drogas, suicídio, mutilação por acidente ou provocada por outro indivíduo. No segundo grupo, com relação aos eventos não intencionais provocados pelo homem, encontram-se incêndios, explosões, queda de pontes e viadutos, acidentes automobilísticos, aéreos e aquáticos e perda de parte do corpo em ambiente de trabalho. No terceiro grupo, entre os desastres naturais, incluem-se tornado, avalanche, erupção vulcânica, ataques de animais, terremoto, furacão, enchentes e epidemias (Schiraldi, 1999).

Dessa maneira, é possível perceber que existe uma gama de situações que podem ser consideradas traumáticas. Segundo a quarta edição revisada do Manual Diagnóstico e Estatístico de Transtornos Mentais (DSM-IV-TR; APA 2002) o evento estressor traumático pode ser definido como uma situação de estresse que foi experimentada, testemunhada ou confrontada, na qual houve ameaça à vida da pessoa ou de alguém próximo a ela. Diante desses eventos, a pessoa reage com medo e desesperança, além de tentar evitar a rememoração da experiência (Sher, 2004).

Hans Selye, no ano de 1930, popularizou o termo estresse ao identificar o seu efeito sobre a saúde física das pessoas. McEwen (2002) conceitua o estresse como a pressão que a vida exerce sobre nós e a forma como essa pressão faz-nos sentir. De acordo com Schneiderman, Ironson e Siegel (2005), em 1956, Selye usou o termo "estresse" para representar os efeitos de qualquer estímulo que ameaçasse seriamente a homeostase, sendo que a ameaça atual percebida pelo organismo foi referida como um "estressor" e a resposta ao estressor foi denominada "resposta de estresse". Além disso, os autores pontuam que, embora respostas de estresse evoluam como um processo adaptativo, respostas de estresse severo e prolongado poderiam levar ao dano e à doença (Schneiderman \& colaboradores, 2005).

Ao se defrontar com um evento estressor, o organismo tende a ter uma resposta de estresse que pode ser aguda e momentânea ou pode ser crônica (McEwen \& Lashley, 2004). Na resposta de estresse agudo, sendo essa definida como um estado transitório de excitação, com início e fim claros, o organismo tenta se adaptar da melhor forma possível ao evento estressor. $\mathrm{Na}$ situação de estresse crônico, ou seja, durante o estado contínuo de ativação em que o indivíduo percebe as demandas do ambiente como sendo superiores aos recursos internos e externos disponíveis, o organismo precisa estar constantemente pronto para a situação de perigo na qual foi ou continua sendo submetido (Gerrig \& Zimbardo, 2005; McEwen \& Lashley, 2004). No TEPT, especificamente, o indivíduo tem uma sensação presente de ameaça (Ehlers \& Clark, 2000), ficando em alerta constante. Dessa maneira, o organismo permanece num estado crônico de estresse, ativando um padrão de respostas para lidar com estímulos no ambiente interpretados como ameaçadores (Yehuda \& LeDoux, 2007).

\section{Desenvolvimento Histórico}

Historicamente, por volta de 1970, psiquiatras americanos começaram a fazer grupos com veteranos da Guerra do Vietnã (Schestatsky \& colaboradores, 2003; Sher, 2004). A partir da difusão desses grupos e da literatura desenvolvida na época, pesquisadores organizaram uma lista dos sintomas mais comuns observados nas "neuroses traumáticas", denominação dada anteriormente para os quadros de estresse póstraumático (Lifton \& Olson, 1976). Nessa época, surgiu a necessidade de um diagnóstico que abarcasse essa categoria de indivíduos previamente saudáveis que desenvolviam reações sintomáticas particulares e persistentes após a vivência de um determinado evento estressor (Kristensen, Parente \& Kaszniak, 2005). Desse modo, foi introduzido na literatura científica os critérios diagnósticos contidos na $3^{\text {a }}$ edição do Manual Diagnóstico e Estatístico dos Transtornos Mentais (DSM-III), publicado pela Associação Psiquiátrica Americana (APA), em 1980 (APA, 1980; Saight \& Bremmer, 1999; Schestatsky e colaboradores, 2003; Schnurr, Friedman \& Bernardy, 2002). Assim, é possível perceber que as edições mais antigas do DSM não continham base empírica, e os critérios específicos eram insuficientes quando se referiam a reações ao estresse (Calhoun \& Resick, 1999).

Para estabelecer o diagnóstico de TEPT, no DSM-III (APA, 1980), era necessária a experiência direta de um evento altamente ameaçador à vida ou à integridade física do indivíduo que fosse severa para produzir sintomas de sofrimento e estivesse fora da cadeia normal de experiências humanas. Além disso, com relação aos sintomas desenvolvidos posteriormente à experiência do evento, os seguintes critérios deveriam estar presentes: no mínimo um sintoma de revivência ou reexperiência, ao menos um sintoma de entorpecimento da responsividade geral e, no mínimo, dois sintomas de uma lista de sintomas inespecíficos (APA, 1980).

$\mathrm{Na}$ terceira edição revisada do DSM (DSM-IIIR; APA, 1987), passou a considerar-se possível o desenvolvimento do TEPT após o indivíduo conhecer ou testemunhar o evento estressor, diferenciando-se da edição anterior, que exigia a experiência direta do evento. Ao mesmo tempo, manteve-se a necessidade de que o evento estressor estivesse fora da cadeia normal de experiências humanas. Apesar disso, evidências sugeriam que eventos comuns na vida das 
pessoas também desencadeavam o transtorno (Schestatsky e colaboradores, 2003).

Assim, posteriormente, foram realizadas pesquisas específicas objetivando reavaliar o TEPT e incluir suas modificações na $4^{\mathrm{a}}$ edição do DSM (DSMIV; APA, 1994). Nessa $4^{a}$ edição, a exposição ao evento estressor foi ampliada, englobando tanto características descritivas do estressor, como respostas subjetivas do indivíduo ao evento (APA; Corcoran, Green, Goodman \& Krinsley, 2000). Alguns critérios das edições anteriores do DSM foram eliminados em função da variabilidade de possíveis respostas entre os indivíduos e também pela característica de que muitos eventos estressores fazem parte da cadeia normal de eventos experienciados (Briere, 1997).

\section{Critérios Diagnósticos e Características Principais}

De acordo com a revisão de texto do DSM-IV (DSM-IV-TR; APA, 2002), o TEPT caracteriza-se por um conjunto de sintomas que se manifesta após a exposição a um evento estressor traumático, incluindo revivência persistente, embotamento relacionado à responsividade geral, esquiva e excitabilidade aumentada. Os sintomas de revivência mais típicos do TEPT manifestam-se na forma de recordações ou pesadelos, que tendem a ser cenas ou aspectos relacionados à experiência traumática (APA, 2002). Quanto à esquiva, essa tende a acontecer pela necessidade que o indivíduo tem de proteger-se do afeto negativo e da excitabilidade associada à revivência da experiência (Calhoun \& Resick, 1999). Autores como Litz (1992) acreditam que o embotamento (também observado como entorpecimento) pode ser descrito como um déficit seletivo do processamento emocional, combinado ou não com medo de perder o controle, visto que a deficiência emocional provoca reatividade elevada aos eventos negativos (Calhoun \& Resick, 1999).

Dessa maneira, assim como eventos traumáticos devem ser diferenciados de outros eventos estressores, o TEPT deve ser diferenciado de outras respostas possíveis (Kristensen e colaboradores, 2005). É importante ter claro que os sintomas apresentados devem estar conectados ao evento estressor traumático (APA, 2002). Para o diagnóstico de TEPT, é necessário que a pessoa tenha vivenciado ou testemunhado um ou mais eventos que envolvam morte ou grave ferimento, reais ou ameaçadores, ou uma ameaça à integridade física própria ou a de outros. O tempo mínimo de sintomatologia exigida para caracterização do transtorno é de um mês após o início do trauma. Conforme o critério F do DSM-IV-TR (APA, 2002), deve-se assegurar que o transtorno acarreta sofrimento significativo e/ ou prejuízo funcional. No entanto, pode ser difícil avaliar criteriosamente esse aspecto. Breslau e Alvarado (2007) realizaram um estudo sobre o impacto desse critério para o diagnóstico de TEPT, alegando a dificuldade de sua avaliação pela amplitude que apresenta. Os achados da pesquisa permitiram concluir que, se o critério de prejuízo clínico significativo não fosse necessário para o diagnóstico de TEPT, as taxas de indivíduos com esse diagnóstico seriam, provavelmente, $30 \%$ mais altas. Por essa razão, percebe-se que ainda há controvérsias sobre quão bem estão descritos os critérios que podem traduzir o TEPT (Breslau \& Alvarado, 2007). No DSM-IV-TR (APA, 2002), é descrito que o TEPT, frequentemente, resulta em prejuízos na habilidade de relacionamento interpessoal em ambientes sociais e familiares, os quais podem ser percebidos devido a fatores como instabilidade no trabalho, problemas conjugais e divórcio, discordância com familiares e amigos e dificuldades em lidar com os filhos. Entretanto, nem sempre é fácil identificar se os prejuízos percebidos são decorrentes do diagnóstico de TEPT ou já estavam presentes anteriormente à experiência do evento estressor, podendo, dessa maneira, ter contribuído para o desencadeamento e a manutenção dos sintomas póstraumáticos.

O TEA foi introduzido pela primeira vez na literatura em 1994, a partir do DSM-IV (APA, 1994), tendo como objetivos principais caracterizar reações graves e de curta duração em até quatro semanas após o trauma e identificar indivíduos que poderiam, subsequentemente, desenvolver TEPT (Harvey \& Bryant, 2002; McNally, 1998). Dessa forma, com a inclusão do diagnóstico de TEA procurou-se suprimir uma lacuna existente no DSM-III-R (APA, 1987) que apontava para a necessidade de uma duração mínima de um mês de sintomas para o diagnóstico de TEPT sem cogitar a importância dos sintomas anteriores a esse tempo (Bryant \& Harvey, 1999).

Entretanto, Marshall, Spitzer e Liebowitz (1999) salientam que a inclusão desse diagnóstico suscitou no aparecimento de algumas divergências, como por exemplo, com relação à ênfase dada aos sintomas dissociativos, à descrição do curso longitudinal do transtorno e à distinção entre respostas patológicas e respostas esperadas após a vivência de um evento estressor traumático. Ao mesmo tempo, os autores se questionam quanto à existência de evidências empíricas suficientes para sustentar ambos os diagnósticos, caso fosse desconsiderada a distinção temporal entre TEA e TEPT.

Apesar disso, o diagnóstico de TEA não deve ser relativizado, uma vez que parece predispor o indivíduo ao TEPT (Birmes e colaboradores, 2003; Brooks e colaboradores, 2008; Harvey \& Bryant, 2000). 
Ao mesmo tempo, há controvérsias com relação a isso, tendo em vista que estudos mostram que pessoas desenvolvem TEPT sem necessariamente passarem pelo diagnóstico de TEA (Bryant, 2003). Portanto, atualmente, há uma discussão que surge a fim de clarificar a ideia de que TEA e TEPT sejam construtos similares ou diferentes, ressaltando para a problemática conceitual observada no TEA (Ballenger e colaboradores, 2004; Harvey \& Bryant, 2002).

Para a $5^{\text {a }}$ edição do DSM algumas modificações conceituais foram propostas em um primeiro esboço do manual (APA, 2010). O conceito de trauma sofreu alterações no critério A1, pois era tido como ambíguo e amplo em algumas situações e restrito em outras, sendo facilmente manipulado em processos judiciais (Friedman, 2010; McNally, 2009). Dessa maneira, na nova proposta, tal critério vem com a intenção de facilitar a distinção entre evento traumático e evento estressor sem características traumáticas (APA, 2010). Ao mesmo tempo, o critério A2 foi excluído após estudos mostrando que ele era dispensável para o diagnóstico de TEPT (McNally, 2009; Brewin e colaboradores, 2009; O’Donnel, Creamer, McFarlane, Silove \& Bryant, 2010; Shalev, 2009). Então, evento traumático seria a pessoa ser exposta ou ameaçada de morte, grave ferimento ou abuso sexual em uma ou mais das seguintes maneiras: (1) experienciando o evento ela mesma; (2) assistindo o evento ocorrendo com outros; (3) recebendo a notícia de que o evento ocorreu com alguém próximo e envolveu morte ou ameaça, de forma violenta ou acidental; (4) experienciando o evento de forma repetida ou de maneira extrema - esta não inclui exposições via mídia eletrônica, televisão, filmes ou fotografias, a menos que essa exposição esteja relacionada com o trabalho. Essas modificações se aplicam tanto para o TEPT como para o TEA, uma vez que ambos necessitam da ocorrência de um evento traumático.

No diagnóstico de TEPT, outras propostas de modificações importantes dizem respeito à divisão do critério $C$ (que incluía sintomas de esquiva e entorpecimento) em dois diferentes critérios. Estudos, como o de Asmundson, Stapleton e Taylor (2004), mostram que os sintomas de esquiva e de entorpecimento não devem ser considerados conjuntamente em um único agrupamento de sintomas. Para o $D S M-V$, propõe-se que o critério de esquiva deve contemplar, no mínimo, um dos seguintes sintomas: evitar lembranças internas como pensamentos e sentimentos e/ou lembranças externas como lugares e conversas. E, para o critério de entorpecimento, provavelmente denominado de "alterações negativas em cognições e humor", foram acrescentados três sintomas aos que já eram descritos na edição anterior do DSM: (1) crenças persistente e exageradamente negativas sobre si, outros e mundo; (2) persistente culpabilização de si ou outros sobre as causas ou as consequências do evento traumático; e (3) emoções negativas invasivas, como raiva, medo, culpa, horror (APA, 2010).

\section{Prevalência}

Sher (2004) aponta que estudos epidemiológicos sobre a prevalência de exposição a eventos estressores traumáticos ao longo da vida encontraram taxas que variaram de $40 \%$ a $90 \%$. Uma possível explicação para a discrepância na taxa de prevalência poderia ser justificada pela definição do que seria considerado como um evento traumático, visto que a prevalência foi se modificando ao longo da reformulação dos critérios diagnósticos. Conforme Kristensen e colaboradores (2005), estudos que utilizaram os critérios do DSM-III (APA, 1980) e do DSM-III-R (APA, 1987) para verificar a prevalência de eventos estressores traumáticos na população geral ao longo da vida encontraram taxas que variaram de $39,1 \%$ a $75 \%$. No estudo de Kessler, Sonnega, Bromet, Hughes e Nelson (1995), utilizando critérios do DSMIII-R, foi encontrada uma prevalência de exposição com uma variação de $60,7 \%$ para homens e de $51,2 \%$ para mulheres que reportaram ao menos um evento traumático. Por outro lado, estudos empregando os critérios do DSM-IV (APA, 1994) encontraram índices sobre a prevalência de eventos estressores que variaram desde $57,1 \%$ a $89,6 \%$ (Kristensen e colaboradores, 2005). Numa pesquisa realizada segundo estes critérios, foi encontrada uma prevalência de eventos de eventos estressores traumáticos em torno de $92,2 \%$ para homens e 87,1\% para mulheres (Breslau \& colaboradores, 1998).

Com relação à prevalência de TEPT, de acordo com Kessler e colaboradores (1995), um grande estudo feito na população geral foi realizado nos Estados Unidos pelo Epidemiologic Catchment Area, cuja prevalência para o transtorno ao longo da vida foi estimada em $1 \%$, segundo os critérios do DSM-III-R. Em outra pesquisa com casos-controle, utilizando esses mesmos critérios, a prevalência ao longo da vida encontrada foi de 2,6\% (Shore, Vollmer, \& Tatum, 1989 citado por Kessler \& colaboradores, 1995).

$\mathrm{Na}$ revisão realizada por Sher (2004), os índices de prevalência de TEPT ao longo da vida variaram de $1 \%$ a $9 \%$. Entre aqueles que utilizaram os critérios do DSM-III-R (APA, 1987), foram encontradas taxas de prevalência do transtorno que variaram de 7,8\% a 9,2\% na população geral (Breslau, Davis, Andreski \& Peterson, 1991; Kessler e 
colaboradores, 1995). O estudo de Kessler e colaboradores encontrou uma distribuição variada de $10,4 \%$ nas mulheres e 5,0\% nos homens. Ademais, Resnick, Kilpatrick, Dansky, Saunders e Best (1993), após investigarem o TEPT em uma amostra de mulheres, encontraram a prevalência estimada ao longo da vida de 12,3\%. Breslau e colaboradores (1991) encontraram, pelos critérios do DSM-III-R (APA, 1987), em uma amostra de adultos jovens, taxas de prevalência de $11,3 \%$ para mulheres e taxas de $6 \%$ para homens. De acordo com Kessler e colaboradores, múltiplos fatores são responsáveis pelas diferenças nos estudos envolvendo o DSM-III (APA, 1980) e o DSMIII-R (APA, 1987), incluindo as diferenças nos critérios diagnósticos, procedimentos de avaliação e outras características das amostras.

Segundo Kristensen e colaboradores (2005), um estudo pioneiro que avaliou a prevalência de TEPT na população geral, considerando os critérios diagnósticos estabelecidos no DSM-IV (APA, 1994), foi o de Breslau e colaboradores (1998). Nesse estudo, os autores verificaram um risco condicional para $\mathrm{O}$ TEPT em 9,2\%, considerando um evento estressor selecionado aleatoriamente e, em torno de 13,6\%, quando o critério de estressor foi baseado no pior evento. No estudo de replicação do National Comorbidity Survey (NCS-R; Kessler, Berglund e colaboradores, 2005; Kessler, Chiu, Demler, Jin \& Walters, 2005), investigou-se a prevalência dos transtornos mentais tanto ao longo da vida como nos últimos 12 meses conforme os critérios descritos no DSM-IV (APA, 1994), estimando uma prevalência de TEPT, nos últimos 12 meses, de 3,5\% nos 9.282 participantes da amostra. Já a prevalência ao longo da vida encontrada para o transtorno foi de $6,8 \%$.

Ainda que a grande maioria dos indivíduos expostos a situações potencialmente traumáticas não desenvolva TEPT (Sher, 2004; Yehuda \& McFarlane, 1995), ele tem sido considerado, entre os transtornos de ansiedade, o terceiro mais prevalente ao longo da vida no estudo NCS-R (Kessler, Berglund e colaboradores, 2005), após Fobia Específica (12,5\%) e Fobia Social (12,1\%). Consequentemente, de forma negativa, o resultado está relacionado a consequências sociais e econômicas bastante significativas (Ballenger e colaboradores, 2004).

\section{Considerações Finais}

Por intermédio da revisão da literatura, fica evidente que esforços ainda vêm sendo empregados numa tentativa de clarificar, dimensionar e mensurar os efeitos do TEPT para aqueles indivíduos acometidos por esse diagnóstico. Ainda há controvérsias entre os estudiosos com relação a alguns aspectos mais particulares e específicos que caracterizam o transtorno, como por exemplo, o tempo mínimo de duração dos sintomas $\mathrm{e}$ as peculiaridades que $\mathrm{o}$ diferenciam do TEA, além das dificuldades em mensurar o sofrimento significativo e/ou o prejuízo funcional observados entre as vítimas.

Dessa maneira, tal indagação sobre quão bem descritos estão os critérios diagnósticos, levando a constantes reformulações nos DSM's, também acarreta em impactos significativos sobre os estudos epidemiológicos de exposição a eventos estressores ao longo da vida e sobre os levantamentos de prevalência do TEPT na população geral. Assim, é possível supor que as peculiaridades observadas no transtorno em diferentes momentos serão marcadas e impactadas pelo contexto histórico e social, influenciando não só nos tipos de eventos estressores traumáticos mais frequentemente experienciados, como também no entendimento e nas estratégias de enfrentamento utilizadas pelos indivíduos que sofrem de TEPT.

Apesar disso, percebe-se certa tendência entre os diferentes estudos que embasaram este artigo de ressaltar a alta prevalência do transtorno e também a ampla gama de prejuízos observados não somente entre as vítimas, mas também entre familiares, amigos e a sociedade como um todo. Em função disso, ressaltase que novos estudos empíricos são importantes, a fim de que estratégias terapêuticas eficientes sejam formuladas, diminuindo a intensidade dos sintomas, aprimorando as estratégias de enfrentamento e, consequentemente, aumentando a qualidade de vida dos pacientes diagnosticados com TEPT.

\section{Referências}

American Psychiatric Association. (1980). Manual diagnóstico e estatístico de transtornos mentais (3a. ed.). Porto Alegre: Artmed.

American Psychiatric Association. (1987). Manual diagnóstico e estatístico de transtornos mentais (3a. ed.; Texto Revisado). Porto Alegre: Artmed.

American Psychiatric Association. (1994). Manual diagnóstico e estatístico de transtornos mentais (4a. ed.). Porto Alegre: Artmed.

American Psychiatric Association. (2002). Manual diagnóstico e estatístico de transtornos mentais (4a. ed.; Texto Revisado). Porto Alegre: Artmed.

American Psychiatric Association. (2010). Proposed draft revisions to DSM Disorders and Criteria. Acesso em 02 de Junbo de 2010, em bttp:/ / www.dsm5.org/

Asmundson, G. J. G., Stapleton, J. A. \& Taylor, S. (2004). Are avoidance and numbing distinct 
PTSD symptom clusters? Journal of Traumutic Stress, 17(6), 467-475.

Ballenger, J. C., Davidson, J. T. R., Lecrubier, Y., Nutt, D. J., Marshall, R. D., Nemeroff, C. B., Shalev, A. Y. \& Yehuda, R. (2004). Consensus statement update on posttraumatic stress disorder from the International Consensus Group on Depression and Anxiety. Journal of Clinical Psychiatry, 65, 55-62.

Birmes, P., Brunet, A., Carreras, D., Ducassé, J. L., Charlet, J. P. \& Lauque, D. (2003). The predictive power of peritraumatic dissociation and acute stress symptoms for posttraumatic stress symptoms: a three-month prospective study. American Journal of Psychiatry, 160, $1337-$ 1339.

Breslau, N. \& Alvarado, G. F. (2007). The clinical significance criterion in DSM-IV posttraumatic stress disorder. Psychological Medicine, 37, 1437-1444.

Breslau, N., Davis, G. C., Andreski, P. \& Peterson, E. (1991). Traumatic events and posttraumatic stress disorder in an urban population of young adults. Archives of General Psychiatry, 48, 216-222.

Breslau, N., Kessler, R. C., Chilcoat, H. D., Schultz, L. R., Davis, G. C. \& Andreski, P. (1998). Trauma and posttraumatic stress disorder in the community: the 1996 Detroit Area Survey of Trauma. Archives of General Psychiatry, 55, 626632.

Brewin, C. R., Lanius, R. A., Novac, A., Schnyder, U. \& Galea, S. (2009). Reformulating PTSD for $D S M-V$ : life after criterion A. Journal of Traumatic Stress, 22(5), 366-373.

Briere, J. (1997). Psychological assessment of adult posttraumatic states. Washington, DC: American Psychological Association.

Brooks, R., Silove, D., Bryant, R., O'Donnell, M., Creamer, M. \& McFarlane, A. (2008). A confirmatory factor analysis of the acute stress disorder interview. Journal of Traumatic Stress, 21, 352-355.

Bryant, R. A. (2003). Early predictors of posttraumatic stress disorder. Biological Psychiatry, 53, 789-795.

Bryant, R. A. \& Harvey, A. G. (1999). Acute stress disorder: a bandbook of theory, assessment, and treatment. Washington, DC: American Psychological Association.

Calhoun, K. S. \& Resick, P. (1999). Transtorno de estresse pós-traumático. Em D. H. Barlow (Org.). Manual dos Transtornos Psicológicos (2a. ed.) (pp. 63-118). Porto Alegre: Artmed.
Corcoran, C. B., Green, B. L., Goodman, L. A. \& Krinsley, K. E. (2000). Conceptual and methodological issues in trauma history assessment. Em A. Y. Shalev, R. Yehuda \& A. C. McFarlane (Orgs.). International handbook of buman response to trauma (pp. 223-232). New York: Kluwer/Plenum.

Ehlers, A. \& Clark, D. M. (2000). A cognitive model of posttraumatic stress disorder. Behaviour Research and Therapy, 38, 319-345.

Friedman, M. J. (2010, May 21). PTSD revisions proposed for DSM-5, with input from array of experts. Psychiatric News, 45(10), 8-33.

Gerrig, R. J. \& Zimbardo, P. G. (2005). A psicologia e a vida (16a. ed.). Porto Alegre: Artmed.

Harvey, A. G. \& Bryant, R. A. (2000). Two-year prospective evaluation of the relationship between acute stress disorder and posttraumatic stress disorder following mild traumatic brain injury. American Journal of Psychiatry, 157, 626-628.

Harvey, A. G. \& Bryant, R. A. (2002). Acute stress disorder: a synthesis and critique. Psychological Bulletin, 128, 886-902.

Kessler, R. C., Berglund, P., Demler, O., Jin, R., Merikangas, K. R. \& Walters, E. E. (2005). Lifetime prevalence and age-of-onset distributions of DSM-IV Disorders in the National Comorbidity Survey Replication. Archives of General Psychiatry, 62, 593-602.

Kessler, R. C., Chiu, W. T., Demler, O., Jin, R. \& Walters, E. E. (2005). Prevalence, severity, and comorbidity of 12-month DSM-IV Disorders in the National Comorbidity Survey Replication. Archives of General Psychiatry, 62, 617-627.

Kessler, R. C., Sonnega, A., Bromet, E., Hughes, M. \& Nelson, C. B. (1995). Posttraumatic stress disorder in the National Comorbidity Survey. Archives of General Psychiatry, 52, 1048-1060.

Kristensen, C. H., Parente, M. A. M. P. \& Kaszniak, A. W. (2005). Transtorno de Estresse PósTraumático: Critérios diagnósticos, prevalência e avaliação. Em R. M. Caminha (Org.). Transtornos do Estresse Pós-Traumático (TEPT): da neurobiologia à terapia cognitiva (pp. 15-35). São Paulo: Casa do Psicólogo.

Lifton, R. J. \& Olson, E. (1976). The human meaning of total disaster: the Buffalo Creek Experience. Psychiatry, 39, 1-18.

Litz, B. T. (1992). Emotional numbing in combatrelated post-traumatic stress disorder: a critical review and reformulation. Clinical Psychology Review, 12, 417-432. 
Marshall, R. D., Spitzer, R. \& Liebowitz, M. R. (1999). Review and critique of the new DSM-IV diagnosis of acute stress disorder. American Journal of Psychiatry, 156, 1677-1685.

McEwen, B. S. (2002). Sex, stress, and the hippocampus: allostasis, allostatic load and the aging process. Neurobiology of Aging, 23, 921939.

McEwen, B. S. \& Lashley, E. N. (2004). The end of stress as we know it. Washington, DC: National Academies Press.

McNally, R. J. (1998). Experimental approaches to cognitive abnormality Posttraumatic Stress Disorder. Clinical Psychology Review, 18, 971-982.

McNally, R. J. (2009). Can we fix PTSD in DSM-V? Depression and Anxiety, 26, 597-600.

O’Donnell, M. L., Creamer, M., McFarlane, A. C., Silove, D. \& Bryant, A. R. (2010). Should A2 be a diagnostic requirement for posttraumatic stress disorder in DSM-V? Psychiatry Research, 176, 257-260.

Resnick, H. S., Kilpatrick, D. G., Dansky, B. S., Saunders, B. E. \& Best, C. L. (1993). Prevalence of civilian trauma and PTSD in a representative national sample of women. Journal of Consulting and Clinical Psychology, 61, 984-991.

Saigh, P. A. \& Bremner, J. (1999). The history of posttraumatic stress disorder. Em P. A. Saigh \& J. Bremner (Orgs.). Posttraumatic Stress Disorder: a comprehensive text (pp. 1-17). Boston: Allyn \& Bacon.

Schestatsky, S., Shansis, F., Ceitlin, L. H., Abreu P. B. S. \& Hauck, S. (2003). A evolução histórica do conceito de estresse pós-traumático. Revista Brasileira de Psiquiatria, 25, 8-11.

Schiraldi, G. R. (1999). The post-traumatic stress disorder sourcebook: a guide to healing, recovery, and growth. New York: Mc Graw Hill.

Schneiderman, N., Ironson, G. \& Siegel, S. D. (2005). Stress and health: psychological, behavioral, and biological determinants. Annual Review of Clinical Psychology, 1, 607-628.

Schnurr, P. P., Friedman, M. J. \& Bernardy, N. C. (2002). Research on Posttraumatic Stress Disorder: epidemiology, pathophysiology, and assessment. Journal of Clinical Psychology, 58, 877889.

Shalev, A. Y. (2009). Posttraumatic Stress Disorder and Stress-Related Disorders. Psychiatric Clinics of North America, 32, 687-704.

Sher, L. (2004). Recognizing post-traumatic stress disorder. QJM: An International Journal of Medicine, 97, 1-5.

Yehuda, R. \& LeDoux, J. (2007). Response Variation following Trauma: A translational neuroscience approach to understanding PTSD. Neuron, 56(1), 19-32.

Yehuda, R. \& McFarlane, A. C. (1995). Conflict between current knowledge about posttraumatic stress disorder and its original conceptual basis. American Journal of Psychiatry, 152, 1705-1713.

Recebido em setembro de 2010

Reformulado em novembro de 2010

Aprovado em dezembro de 2010

Sobre os autores:

Gabriela Sbardelloto é Psicóloga (PUCRS). Especialista em Psicologia Clínica - ênfase em Avaliação Psicológica (UFRGS) e especialista em Psicologia Clínica - ênfase em Psicologia Cognitivo-Comportamental (Clínica WP). Mestre em Psicologia/Cognição Humana (PUCRS). Pesquisadora do Grupo de Pesquisa "Cognição, Emoção e Comportamento" do PPG em Psicologia da PUCRS.

Luiziana Souto Schaefer é Psicóloga (PUCRS). Perita Criminal Psicóloga do Instituto-Geral de Perícias do Rio Grande do Sul (IGP-RS). Mestranda em Psicologia/Cognição Humana (CNPq-PUCRS). Pesquisadora do Grupo de Pesquisa "Cognição, Emoção e Comportamento" do PPG em Psicologia da PUCRS.

Alice Reuwsaat Justo é Acadêmica da Faculdade de Psicologia (PUCRS). Integrante do Grupo de Pesquisa “Cognição, Emoção e Comportamento” do PPG em Psicologia da PUCRS.

Christian Haag Kristensen é Psicólogo (PUCRS). Especialista em Neuropsicologia (CRP/07). Formação em Terapia Cognitiva pelo Beck Institute for Cognitive Therapy and Research. Mestre e Doutor em Psicologia (UFRGS). Coordenador do Grupo de Pesquisa "Cognição, Emoção e Comportamento" e Professor do PPG em Psicologia da PUCRS. 